\title{
LOCATION OF THE ZEROS OF A POLYNOMIAL RELATIVE TO CERTAIN DISKS
}

BY

\author{
R. C. RIDDELL
}

ABSTRACT. The zeros of the complex polynomial $P(z)=z^{n}+\Sigma \alpha_{i} z^{n-i}$ are studied under the assumption that some $\left|\alpha_{k}\right|$ is large in comparison with the other $\left|\alpha_{i}\right|$. It is shown under certain conditions that $P(z)$ has $n-k$ zeros in $|z|<m_{-}$ and $k$ zeros in $|z|>m_{+}$, where $m_{-}<m_{+}<\left|\alpha_{k}\right|^{1 / k}$; and under suitably strengthened conditions, one of the $k$ zeros of larger modulus is shown to lie in each of the $k$ disks $\mid z-\left(-\alpha_{k}\right)^{1 / k_{1}}<R$, where $m_{-}+R<\left|\alpha_{k}\right|^{1 / k}$.

1. Notation and basic estimate. If the complex polynomial

$$
P(z)=z^{n}+\alpha_{1} z^{n-1}+\cdots+\alpha_{n-1} z+\alpha_{n}
$$

has a dominant coefficient, in the sense that some $\left|\alpha_{k}\right|$ is large in comparison with the other $\left|\alpha_{i}\right|$, then $P(z)$ has $n-k$ zeros near 0 and one zero near each of the $k$ values of $\left(-\alpha_{k}\right)^{1 / k}$. We shall establish some conditions under which precise estimates can be given. The first type (Estimate $A$ in $\$ 2$ below), which goes back in principle to a theorem of Pellet $[3$, p. 393], [1, p. 10], asserts the existence of a zero-free annulus $m_{-}<|z|<m_{+}$. The second type (Estimate $B$ in \$3) further asserts, under stronger conditions, the existence of $k$ disks $\left|z-\left(-\alpha_{k}\right)^{1 / k}\right| \leqslant R$, each one of which isolates a single zero of $P(z)$.

These results rest on the following simple observation. Let $k$ denote an integer in the range $1 \leqslant k \leqslant n$, chosen now and fixed in the sequel. With $P(z)$ as above, suppose that $P(z)=0$ and $z \neq 0$. Transposing all terms other than $z^{n}+\alpha_{k} z^{n-k}$, dividing by $z^{n-k}$, and applying the triangle inequality, we obtain

$$
\left|z^{k}+\alpha_{k}\right| \leqslant \sum_{i \neq k}\left|\alpha_{i}\right||z|^{k-i}
$$

For convenience in estimating the right side, we define

$$
\begin{aligned}
a & =\left|\alpha_{k}\right|, \quad b=\left|\alpha_{1}\right|+\cdots+\left|\alpha_{k-1}\right|, \quad c=\left|\alpha_{k+1}\right|+\cdots+\left|\alpha_{n}\right|, \\
g(r) & =\left\{\begin{array}{lc}
b r+c r^{k-n} & (0<r \leqslant 1), \\
b r^{k-1}+c r^{-1} & (r \geqslant 1),
\end{array}\right.
\end{aligned}
$$

Received by the editors June 21, 1972.

AMS (MOS) subject classifications (1970). Primary 30A08, 26A78.

Key words and phrases. Dominant coefficient, zero-free annulus, disk which isolates a single zero. 
where it is understood that $b=0$ in case $k=1$, and $c=0$ in case $k=n$. Then as an immediate consequence of the above inequality, we obtain our basic estimate:

LEMma 1. If $P(z)=0$ and $|z|>0$, then

$$
\left|z^{k}+\alpha_{k}\right| \leqslant g(|z|) \text {. }
$$

In what follows, the quantities $P(z), k, a, b, c$, and $g(r)$ will continue to have the meanings given above.

2. Annuli which contain no zero. The conclusions established in this section all have the same form, which we state in advance for ease of reference.

Estimate A. $P(z)$ has $n-k$ zeros in the disk $|z| \leqslant m_{-}$and $k$ zeros in the region $|z| \geqslant m_{+}$, where $m_{-}<m_{+} \leqslant a^{1 / k}$.

Here of course zeros are being counted with their multiplicities. Theorems asserting the validity of Estimate $A$ are deduced below from the following consequence of Lemma 1.

LEMMA 2. Estimate A holds if $r=m_{-}$and $r=m_{+}$are two solutions of a relation of the form

$$
a=h(r), \text { where } h(r) \geqslant r^{k}+g(r) .
$$

PROOF. If $P(z)=0$ and $r=|z|>0$, then by applying (1) to the inequality $\left|\alpha_{k}\right| \leqslant\left|-z^{k}\right|+\left|z^{k}+\alpha_{k}\right|$ we see that $a \leqslant r^{k}+g(r)$. By the convexity of $r^{k}+g(r)$, the last inequality fails to hold for $r$ in any interval of the form $m_{-}<r<m_{+}$with $m_{ \pm}$solutions of relation (2). Thus if such $m_{ \pm}$exist, the zeros of $P(z)$ are confined to the union of the two separate domains $|z| \leqslant m_{-}$and $|z| \geqslant m_{+}$, which we may label $I$ and $E$ respectively.

If $P_{t}(z)$ is the polynomial obtained by multiplying each coefficient $\alpha_{i}$ of $P(z)$ other than $\alpha_{k}$ by a parameter $t, 0 \leqslant t \leqslant 1$, then the corresponding $g_{t}(r)$ is dominated by $g(r)$ for every $r>0$. Hence the zeros $z_{i}(t)$ of $P_{t}(z)$ also belong to $I \cup E$. But the $z_{i}(t)$ are continuous in $t$, and since precisely $n-k$ of them are in the connected component $I$ when $t=0$, the same must be true when $t=1$. Q.E.D.

Lemma 2 is essentially the above-mentioned result of Pellet, adapted to the present context. Our first application of it gives conditions for the existence of $m_{ \pm}$to the right of $r=1$.

TheOREM 2.1. Assume $1<a \leqslant 1+b+c$, and $D>0$, where

$$
D=\frac{1}{4}\left(a^{1 / k}+b\right)^{2}-\frac{a^{1 / k}-1}{a-1}(a b+c) .
$$


Then Estimate A holds with

$$
m_{ \pm}=1 / 2\left(a^{1 / k}-b\right) \pm D^{1 / 2} .
$$

Proof. Multiplying the equation $a=r^{k}+g(r)$ for $r \geqslant 1$ by $r$, we obtain an equation equivalent to the pair

$$
\begin{aligned}
(s-a)(r+b) & =-(a b+c), \\
s & =r^{k} .
\end{aligned}
$$

Over the interval $1 \leqslant r \leqslant a^{1 / k}$, the convex curve (5) lies on or below the chord $C$ joining the points $(1,1)$ and $\left(a^{1 / k}, a\right)$. Substitution of the equation of $C$ into (4) leads to a quadratic in $r$, whose solutions are the $m_{ \pm}$of (3).

It is clear from (3) that $m_{-}<m_{+}<a^{1 / k}$, and one finds that $m_{-} \geqslant 1$ is equivalent to

$$
\frac{a^{1 / k}-1}{a-1}(1+b+c-a) \geqslant 0,
$$

which holds under the present assumptions. Thus $m_{ \pm}$belong to the interval $1 \leqslant r \leqslant a^{1 / k}$ where the chord $C$ lies on or above (5), so that the quadratic which they satisfy is an equation of the type (2), and Lemma 2 applies. Q.E.D.

Next we deal with a case in which $m_{-}$and $m_{+}$lie on opposite sides of $r=1$.

TheOREM 2.2. Assume $1+b+c<a$. Then Estimate A holds with

$$
\begin{aligned}
& m_{-}=(c /(a-b-1))^{1 /(n-k)}, \\
& m_{+}=((a-c) /(b+1))^{1 / k},
\end{aligned}
$$

and also with $m_{-}$given by (6) and $m_{+}$by (3).

Proof. The function $r^{k}+g(r)$ is everywhere dominated by

$$
h(r)= \begin{cases}1+b+c r^{k-n} & (r \leqslant 1) \\ (1+b) r^{k}+c & (r \geqslant 1)\end{cases}
$$

Thus the equation $a=h(r)$, which has solutions $m_{ \pm}$as in (6) and (7) under the present assumptions, is of the form (2), and Lemma 2 applies.

Over the interval $1 \leqslant r \leqslant a^{1 / k}$, we can also dominate $s=r^{k}$ by the chord used in the previous proof, and so the present theorem remains true with $m_{+}$given by (3) instead of (7). Q.E.D.

The case $b=0$ of Theorem 2.2 strengthens a result of Parodi [2, pp. 139140]. 
It remains to consider the possibility that $m_{ \pm}$are both to the left of $r=$ 1:

THEOREM 2.3. Assume $c<a \leqslant 1+b+c$, and $b+2 d^{1 / 2}<a$, where

$$
d \geqslant \begin{cases}a(c / a)^{k /(n-k)} & \text { if } k<1 / 2 n, \\ \min \left\{1, a^{2-n / k}\right\} c & \text { if } k \geqslant 1 / 2 n .\end{cases}
$$

Then Estimate A holds with

$$
m_{ \pm}=\left\{\frac{1}{2}(a-b) \pm\left[\frac{1}{4}(a-b)^{2}-d\right]^{1 / 2}\right\}^{1 / k}
$$

Proof. Let $r_{*}$ be the solution of $c r_{*}^{k-n}=a$, or $r_{*}=0$ in case $c=0$, and consider the interval

$$
r_{*} \leqslant r \leqslant \min \left\{1, a^{1 / k}\right\}
$$

In this interval we have $b r \leqslant b$, and $c r^{k-n} \leqslant d r^{-k}$ by the definition of $d$ in the statement of the theorem. Hence $b+d r^{-k} \geqslant g(r)$ over the interval (9), and the equation

$$
a=r^{k}+b+d r^{-k}
$$

is of the type (2) there.

The solutions of (10) are just the $m_{ \pm}$of (8), and it is clear from this formula that $m_{-}^{k}<m_{+}^{k} \leqslant a$. The condition $m_{+}^{k} \leqslant 1$ is readily seen to be equivalent to $a \leqslant 1+b+d$, which obviously holds if $a \leqslant 1$ and follows from $a \leqslant 1+\dot{b}+c, c \leqslant d$, in case $a>1$. On the other hand, $m_{ \pm}$as solutions of (10) must exceed the solution $r$ of $a=d r^{-k}$, which in turn is at least $r_{*}$ by the definition of $d$. Thus $m_{ \pm}$belong to the interval (9), where their equation (10) is of the form (2), and so Lemma 2 applies. Q.E.D.

3. Disks which contain a single zero. The conclusions established in this section are all of the following form:

Estimate B. $P(z)$ has $n-k$ zeros in the disk $|z| \leqslant m_{-}$and one zero in each of the $k$ disjoint disks $\left|z-\left(-\alpha_{k}\right)^{1 / k}\right| \leqslant R$, where $m_{-}+R<a^{1 / k}$ $\left(a=\left|\alpha_{k}\right|\right)$.

Again we begin by establishing a preliminary consequence of Lemma 1.

Lemma 3. Suppose that Estimate A holds with $m_{ \pm}$given as in Lemma 2. Suppose that, for some upper bound $M$ on the moduli of the zeros of $P(z)$,

$$
g(M) \leqslant a-m_{+}^{k} \text {. }
$$

Then Estimate B holds with the given $m_{-}$and with 


$$
R=a^{1 / k}-m_{+},
$$

provided also that, in case $k \geqslant 3$,

$$
R<a^{1 / k} \sin (\pi / k) .
$$

Proof. Under the present conditions, the $k$ zeros of $P(z)$ of largest modulus satisfy $m_{+} \leqslant|z| \leqslant M$, and so by inequality (1) of Lemma 1 they must lie in the region

$$
\left|z^{k}+\alpha_{k}\right| \leqslant \max \left\{g(r): m_{+} \leqslant r \leqslant M\right\} .
$$

Since $g$ is a convex function, this maximum is in fact $\max \left\{g\left(m_{+}\right), g(M)\right\}$. But the definition of $m_{+}$by means of inequality (2) implies that $g\left(m_{+}\right) \leqslant$ $a-m_{+}^{k}$, and $g(M)$ admits the same bound by assumption (11). Hence the $k$ zeros of $P(z)$ of largest modulus lie in $\left|z^{k}+\alpha_{k}\right| \leqslant S=a-m_{+}^{k}$.

We claim that this region is covered by the union of $k$ closed disks centered at the $k$ values of $\left(-\alpha_{k}\right)^{1 / k}$, with common radius $R=a^{1 / k}-(a-S)^{1 / k}=$ $a^{1 / k}-m_{+}$, i.e., the $R$ of (12). To see this, it is enough to consider the case $\alpha_{k}=-1$, for the general case follows from it by a dilation and a rotation. Any $z$ satisfying $\left|z^{k}-1\right| \leqslant S$ can be written

$$
z=w\left(1+t e^{i u}\right)^{1 / k} \quad(0 \leqslant t \leqslant S, 0 \leqslant u \leqslant 2 \pi),
$$

where $w^{k}=1$ and the $k$ th root is taken with its principal value. Following a suggestion by D. Boyd, we expand with the binomial series, estimate by the triangle inequality, and observe that the binomial coefficients alternate in sign, to obtain

$$
\begin{aligned}
|z-w| & \leqslant \sum_{m>1}(-1)^{m+1}\left(\begin{array}{c}
1 / k \\
m
\end{array}\right) t^{m} \\
& =1-(1-t)^{1 / k} \leqslant 1-(1-S)^{1 / k},
\end{aligned}
$$

and the claim is verified.

The covering disks obviously do not meet if $k=2$, while in case $k \geqslant 3$ their pairwise disjointness is ensured by assumption (13). Since $m_{-}+R<$ $m_{+}+R=a^{1 / k}$, each of these disks is disjoint from $|z| \leqslant m_{-}$; and a continuity argument similar to the one in the proof of Lemma 2 shows that each one of them contains precisely one zero of $P(z)$. Q.E.D.

Our first result derived from this lemma applies, in case $k \geqslant 2$, only to lacunary polynomials $P(z)$ :

TheOREM 3.1. Assume $a>1, b=0$, and $D>0$, where 


$$
D=\frac{1}{4} a^{2 / k}-\frac{a^{1 / k}-1}{a-1} c .
$$

Then Estimate B holds, with

$$
\begin{aligned}
& m_{-}= \begin{cases}1 / 2 a^{1 / k}-D^{1 / 2} & \text { if } a \leqslant 1+c, \\
(c /(a-1))^{1 /(n-k)} & \text { if } a>1+c,\end{cases} \\
& R=1 / 2 a^{1 / k}-D^{1 / 2},
\end{aligned}
$$

provided also that, in case $k \geqslant 3$,

$$
R<a^{1 / k} \sin (\pi / k)
$$

Proof. With the present assumptions we can apply Theorem 2.1 if $a \leqslant$ $1+c$ and Theorem 2.2 if $a>1+c$, to establish Estimate $A$ with $m_{-}$given by (3) or (6) and $m_{+}$given by (3). The theorem then follows from Lemma 3 as soon as assumption (11) is established. Let $M$ be any upper bound on the moduli of the zeros of $P(z)$. Then $M \geqslant m_{+}$, and since the assumption that $b=0$ makes $g$ a decreasing function, we have $g(M) \leqslant g\left(m_{+}\right)$, from which (11) follows as before. Q.E.D.

The case $k=1$ of Theorem 3.1 , in which the assumption $b=0$ is redundant, refines an estimate due to Parodi [2, pp. 76, 77].

With only a slight loss in precision for small values of $k$, the next result avoids the restriction to lacunary polynomials.

THEOREM 3.2. Assume $1+2 b<\min \left\{a^{1 / k}, a+b-c\right\}$. Then Estimate B holds, with

$$
m_{-}=\left(\frac{c}{a-b-1}\right)^{1 /(n-k)}, \quad R=a^{1 / k}-\left(a-\frac{a b+c}{1+b}\right)^{1 / k}
$$

provided also that, in case $k \geqslant 3, R<a^{1 / k} \sin (\pi / k)$.

ProOF. Since the assumption implies $1+b+c<a$, Theorem 2.2 establishes Estimate A with $m_{-}$given by (6) and $m_{+}$by (7), i.e. by

$$
m_{+}^{k}=(a-c) /(1+b)=a-(a b+c) /(1+b)
$$

Thus the theorem will follow from Lemma 3 as soon as assumption (11) is verified.

To that end, we observe that if $P(z)=0$ and $r=|z|>0$, then (1) and the 
general inequality $\left|z^{k}\right| \leqslant \vdash-\alpha_{k}|+| z^{k}+\alpha_{k} \mid$ together imply that $r^{k} \leqslant a+g(r)$. But the ratio of $a+g(r)$ to $r^{k}$ is a combination of negative powers of $r$, and hence decreases steadily from $+\infty$ to 0 as $r$ increases from 0 . Thus there is precisely one value $r=r_{0}>0$ for which this ratio is unity, i.e. for which

$$
r^{k}=a+g(r)
$$

and according to the observation above, $|z| \leqslant r_{0}$ if $P(z)=0$. To summarize, we may say that $M$ is an upper bound on the moduli of the zeros of $P(z)$ if $M$ exceeds the solution $r_{0}$ of (15), and that this condition $M \geqslant r_{0}$ is equivalent to

$$
g(M) \leqslant M^{k}-a
$$

In the present situation, with $a+b+c>1$, the solution $r_{0}$ of (15) exceeds 1 , and in this region (15) is equivalent to the pair of equations

$$
\begin{aligned}
(s-a)(r-b) & =a b+c, \\
s & =r^{k} .
\end{aligned}
$$

Since $b<a^{1 / k}$ by assumption, the point

$$
(r, s)=\left(a^{1 / k}, a+(a b+c) /\left(a^{1 / k}-b\right)\right)
$$

on the curve (17) lies above the curve (18). But (17) decreases and (18) increases with increasing $r$, and so the point (19) lies above the unique point $\left(r_{0}, r_{0}^{k}\right)$ with $r_{0}>1$ where (17) and (18) intersect. Thus $s>r_{0}^{k}$ and we may take $M=s^{1 / k}$ with the $s$ of (19). By (16), and in view of the assumption that $1+b<a^{1 / k}-b$, this $M$ satisfies

$$
g(M) \leqslant \frac{a b+c}{a^{1 / k}-b} \leqslant \frac{a b+c}{1+b},
$$

which by (14) is the desired inequality (11). Q.E.D.

The two preceding estimates apply when $a>1$. Our final result applies to the complementary case.

THEOREM 3.3. Assume $c<a^{n / k} \leqslant 1$ and $b+2 d^{1 / 2}<a$, and assume $b^{2} \leqslant d$ if $a+b+c>1$, where

$$
d= \begin{cases}a(c / a)^{k /(n-k)} & \text { if } k<1 / 2 n, \\ c & \text { if } k \geqslant 1 / 2 n .\end{cases}
$$


Then Estimate B holds, with

$$
\begin{aligned}
m_{L} & =\left\{1 / 2(a-b)-\left[1 / 4(a-b)^{2}-d\right]^{1 / 2}\right\}^{1 / k}, \\
R & =a^{1 / k}-(a-b-2 d /(a-b))^{1 / k},
\end{aligned}
$$

provided also that, in case $k \geqslant 3, R<a^{1 / k} \sin (\pi / k)$.

Proof. The assumptions imply those of Theorem 2.3, and so Estimate A holds with

$$
m_{ \pm}=\left\{1 / 2(a-b) \pm\left[1 / 4(a-b)^{2}-d\right]^{1 / 2}\right\}^{1 / k} .
$$

Since the square root can be estimated as

$$
\left[1 / 4(a-b)^{2}-d\right]^{1 / 2} \geqslant 1 / 2(a-b)-2 d /(a-b)>0,
$$

Estimate A holds a fortiori with the given $m_{-}$and with $m_{+}>m_{-}$given by

$$
m_{+}^{k}=a-b-2 d /(a-b) \text {. }
$$

Thus the theorem will follow from Lemma 3 as soon as assumption (11) can be verified.

The present assumptions imply that $b<a^{1 / k}$, so that in case $a+b+c>$ 1 we can use the upper bound $M$ of the previous proof. According to (20), this $M$ satisfies $g(M)<(a b+c) /\left(a^{1 / k}-b\right)$. But $a \leqslant 1$ makes $a \leqslant a^{1 / k}$, $c<a$ makes $c \leqslant d$, and $b^{2} \leqslant d$ holds in the present case by assumption. Hence

$$
g(M) \leqslant \frac{a b+c}{a-b}=b+\frac{c+b^{2}}{a-b} \leqslant b+\frac{2 d}{a-b},
$$

and in view of (21), this establishes (11) in case $a+b+c>1$.

If on the other hand $a+b+c \leqslant 1$, then the solution $r_{0}$ of equation (15) in the previous proof lies to the left of 1 ; and we claim in this case that an upper bound $M \geqslant r_{0}$ is defined by

$$
M^{k}=a+b+d^{\prime} /(a+b)
$$

where

$$
d^{\prime}= \begin{cases}a^{2-n / k} c & \text { if } k<1 / 2 n, \\ c & \text { if } k \geqslant 1 / 2 n .\end{cases}
$$

Indeed, if $M^{k} \geqslant 1$ there is nothing to prove. Otherwise $M$ lies in the interval $(a+b)^{1 / k} \leqslant r \leqslant 1$, where $b M \leqslant b$ and $c M^{k-n} \leqslant d^{\prime} M^{-k} \leqslant d^{\prime} /(a+b)$. Then we obtain 


$$
g(M)=b M+c M^{k-n} \leqslant b+d^{\prime} /(a+b)=M^{k}-a
$$

by (22), and according to (16) and the remark above it, our claim is proved. Moreover, by (23), $d^{\prime}=d$ if $k \geqslant 1 / 2 n$, while if $k<1 / 2 n$ one checks that $d^{\prime}<$ $d$ is equivalent to the inequality $c \leqslant a^{n / k}$, which holds by assumption. Thus $d^{\prime} \leqslant d$ for any choice of $k$, and comparing (24) with (21), we conclude that inequality (11) holds as well in case $a+b+c \leqslant 1$. Q.E.D.

If $k=n$ and $a+b>1$, Theorem 3.3 makes no assertion, since here $d=c=0$ and the extra hypothesis $b^{2} \leqslant d$ cannot be met. However, if we assume $b<1 / 2 a$, then Estimate A holds with

$$
m_{+}=a-a b /(a-b)>0
$$

instead of (21), and inequality (11) can be verified just as in the proof above. Thus in the case $k=n, a+b>1$, we may replace the assumptions of Theorem 3.3 by $2 b<a \leqslant 1$ and retain the conclusion with

$$
R=a^{1 / n}-(a-a b /(a-b))^{1 / n} .
$$

\section{REFERENCES}

[1] J. Dieudonné, La théorie analytique des polynomes d'une variable, Mémor. Sci. Math. 93 (1938), 1-71.

[2] M. Parodi, La localisation des valeurs caractéristiques des matrices et ses applications, Gauthier-Villars, Paris, 1959. MR 22 \#1587.

[3] M. Pellet, Sur une mode de séparation des racines des équations et la formule de Lagrange, Bull. Sci. Math. Astronom. 5 (1881), 393-395.

DEPARTMENT OF MATHEMATICS, UNIVERSITY OF BRITISH COLUMBIA, VANCOUVER 8, BRITISH COLUMBIA, CANADA 